\title{
The constrained multiple-sets split feasibility problem and its projection algorithms
}

\author{
Yaqin Zheng ${ }^{1}$, Jinwei Shi ${ }^{2 *}$ and Yeong-Cheng Liou ${ }^{3}$
}

${ }^{\text {*Correspondence: }}$

jinwei_shi@yahoo.com.cn

${ }^{2}$ North China Electric Power University, Baoding, 071003, China

Full list of author information is

available at the end of the article

\begin{abstract}
The projection algorithms for solving the constrained multiple-sets split feasibility problem are presented. The strong convergence results of the algorithms are given under some mild conditions. Especially, the minimum norm solution of the constrained multiple-sets split feasibility problem can be found.
\end{abstract}

\section{Introduction}

Let $H_{1}$ and $H_{2}$ be two real Hilbert spaces. Let $C_{1}, C_{2}, \ldots, C_{N}$ be $N$ nonempty closed convex subsets of $H_{1}$ and let $Q_{1}, Q_{2}, \ldots, Q_{M}$ be $M$ nonempty closed convex subsets of $H_{2}$. Let $A: H_{1} \rightarrow H_{2}$ be a bounded linear operator. The multiple-sets split feasibility problem is formulated as follows:

$$
\text { Find an } x \in \bigcap_{i=1}^{N} C_{i} \text { such that } A x \in \bigcap_{j=1}^{M} Q_{j}
$$

A special case If $N=M=1$, then the multiple-sets split feasibility problem is reduced to the split feasibility problem which is formulated as finding a point $x$ with the property

$$
x \in C \text { and } A x \in Q \text {. }
$$

The split feasibility problem in finite-dimensional Hilbert spaces was first introduced by Censor and Elfving [1] for modeling inverse problems which arise from phase retrievals and in medical image reconstruction [2]. It has been found that the multiple-sets split feasibility problem and the split feasibility problem can be used to model the intensitymodulated radiation therapy [3-6]. Various algorithms have been invented to solve the multiple-sets split feasibility problem and the split feasibility problem, see, e.g., [7-24] and references therein.

The popular algorithm that solves the multiple-sets split feasibility problem and the split feasibility problem is Byrne's CQ algorithm [11] which is found to be a gradient-projection method in convex minimization. Motivated by this idea, in this paper, we present the composite projection algorithms for solving the constrained multiple-sets split feasibility problem. The strong convergence results of the algorithms are given under some mild conditions. Especially, the minimum norm solution of the constrained multiple-sets split feasibility problem can be found.

(c) 2013 Zheng et al.; licensee Springer. This is an Open Access article distributed under the terms of the Creative Commons Attribution License (http://creativecommons.org/licenses/by/2.0), which permits unrestricted use, distribution, and reproduction in any medium, provided the original work is properly cited. 


\section{Preliminaries}

\subsection{Concepts}

Let $H$ be a real Hilbert space with the inner product $\langle\cdot, \cdot\rangle$ and the norm $\|\cdot\|$, respectively, and let $\Omega$ be a nonempty closed convex subset of $H$. Recall that the (nearest point or metric) projection from $H$ onto $\Omega$, denoted by $P_{\Omega}$, is defined in such a way that, for each $x \in H, P_{\Omega}(x)$ is the unique point in $\Omega$ with the property

$$
\left\|x-P_{\Omega}(x)\right\|=\min \{\|x-y\|: y \in \Omega\} .
$$

It is known that $P_{\Omega}$ satisfies

$$
\left\langle x-y, P_{\Omega}(x)-P_{\Omega}(y)\right| \geq\left\|P_{\Omega}(x)-P_{\Omega}(y)\right\|^{2}, \quad \forall x, y \in H .
$$

Moreover, $P_{\Omega}$ is characterized by the following properties:

$$
\left\langle x-P_{\Omega}(x), y-P_{\Omega}(x)\right\rangle \leq 0
$$

for all $x \in H$ and $y \in \Omega$.

We also recall that a mapping $f: \Omega \rightarrow H$ is said to be $\rho$-contractive if $\|T x-T y\| \leq \rho \| x-$ $y \|$ for some constant $\rho \in[0,1)$ and for all $x, y \in \Omega$. A mapping $T: \Omega \rightarrow \Omega$ is said to be nonexpansive if $\|T x-T y\| \leq\|x-y\|$ for all $x, y \in \Omega$. A mapping $T$ is called averaged if $T=(1-\delta) I+\delta U$, where $\delta \in(0,1)$ and $U: \Omega \rightarrow \Omega$ is nonexpansive. In this case, we also say that $T$ is $\delta$-averaged. A bounded linear operator $B$ is said to be strongly positive on $H$ if there exists a constant $\alpha>0$ such that

$$
\langle B x, x\rangle \geq \alpha\|x\|^{2}, \quad \forall x \in H .
$$

Let $A$ be an operator with domain $D(A)$ and range $R(A)$ in $H$.

(i) $A$ is monotone if for all $x, y \in D(A)$,

$$
\langle A x-A y, x-y\rangle \geq 0 .
$$

(ii) Given a number $v>0, A$ is said to be $v$-inverse strongly monotone ( $v$-ism) (or co-coercive) if

$$
\langle A x-A y, x-y\rangle \geq v\|A x-A y\|^{2}, \quad x, y \in H .
$$

It is easily seen that a projection $P_{\Omega}$ is a 1 -ism and hence $P_{\Omega}$ is $\frac{1}{2}$-averaged.

We will need to use the following notation:

- Fix $(T)$ stands for the set of fixed points of $T$;

- $x_{n} \rightarrow x$ stands for the weak convergence of $\left\{x_{n}\right\}$ to $x$;

- $x_{n} \rightarrow x$ stands for the strong convergence of $\left\{x_{n}\right\}$ to $x$.

\subsection{Mathematical model}

Now, we consider the mathematical model of the multiple-sets split feasibility problem. Let $x \in C_{1}$. Assume that $A x \in Q_{1}$. Then we get $\left(I-P_{Q_{1}}\right) A x=0$, which implies $\gamma A^{*}(I-$ 
$\left.P_{Q_{1}}\right) A x=0$, hence $x$ satisfies the fixed point equation $x=\left(I-\gamma A^{*}\left(I-P_{Q_{1}}\right) A\right) x$. At the same time, note that $x \in C_{1}$. Thus,

$$
x=P_{C_{1}}\left(I-\gamma A^{*}\left(I-P_{Q_{1}}\right) A\right) x .
$$

Now, we know $x$ solves the split feasibility problem if and only if $x$ solves the above fixed point equation. This result reminds us that the multiple-sets split feasibility problem is equivalent to a common fixed point problem of finitely many nonexpansive mappings. On the other hand, $x$ solves the multiple-sets split feasibility problem implies that $x$ satisfies two properties:

(i) the distance from $x$ to each $C_{i}$ is zero and

(ii) the distance from $A x$ to each $Q_{j}$ is also zero.

First, we consider the following proximity function:

$$
g(x)=\frac{1}{2} \sum_{i=1}^{N} \alpha_{i}\left\|x-P_{C_{i}} x\right\|^{2}+\frac{1}{2} \sum_{j=1}^{M} \beta_{j}\left\|A x-P_{Q_{j}} A x\right\|^{2},
$$

where $\left\{\alpha_{i}\right\}$ and $\left\{\beta_{j}\right\}$ are positive real numbers, and $P_{C_{i}}$ and $P_{Q_{j}}$ are the metric projections onto $C_{i}$ and $Q_{j}$, respectively. It is clear that the proximity function $g$ is convex and differentiable with the gradient

$$
\nabla g(x)=\sum_{i=1}^{N} \alpha_{i}\left(I-P_{C_{i}}\right) x+\sum_{j=1}^{M} \beta_{j} A^{*}\left(I-P_{Q_{j}}\right) A x .
$$

We can check that the gradient $\nabla g(x)$ is $L$-Lipschitz continuous with constant

$$
L=\sum_{i=1}^{N} \alpha_{i}+\sum_{j=1}^{M} \beta_{j}\|A\|^{2} .
$$

Note that $x^{*}$ is a solution of the multiple-sets split feasibility problem (1.1) if and only if $g\left(x^{*}\right)=0$. Since $g(x) \geq 0$ for all $x \in H_{1}$, a solution of the multiple-sets split feasibility problem (1.1) is a minimizer of $g$ over any closed convex subset, with minimum value of zero. This motivates us to consider the following minimization problem:

$$
\min _{x \in \Omega} g(x)
$$

where $\Omega$ is a closed convex subset of $H_{1}$ whose intersection with the solution set of the multiple-sets split feasibility problem is nonempty, and get a solution of the so-called constrained multiple-sets split feasibility problem

$$
x^{*} \in \Omega \text { such that } x^{*} \text { solves (1.1). }
$$

\subsection{The well-known lemmas}

The following lemmas will be helpful for our main results in the next section.

Lemma 2.1 [25] Let $\left\{x_{n}\right\}$ and $\left\{z_{n}\right\}$ be bounded sequences in a Banach space $X$ and let $\left\{\beta_{n}\right\}$ be a sequence in $[0,1]$ with $0<\liminf _{n \rightarrow \infty} \beta_{n} \leq \limsup _{n \rightarrow \infty} \beta_{n}<1$. Suppose that $x_{n+1}=$ 
$\left(1-\beta_{n}\right) z_{n}+\beta_{n} x_{n}$ for all integers $n \geq 0$ and $\lim \sup _{n \rightarrow \infty}\left(\left\|z_{n+1}-z_{n}\right\|-\left\|x_{n+1}-x_{n}\right\|\right) \leq 0$. Then $\lim _{n \rightarrow \infty}\left\|z_{n}-x_{n}\right\|=0$.

Lemma 2.2 [26] Let $K$ be a nonempty closed convex subset of a real Hilbert space $H$. Let $T: K \rightarrow K$ be a nonexpansive mapping with $\operatorname{Fix}(T) \neq \emptyset$. Then $T$ is demiclosed on $K$, i.e., if $x_{n} \rightarrow x \in K$ weakly and $x_{n}-T x_{n} \rightarrow 0$, then $x=T x$.

Lemma 2.3 [27] Assume that $\left\{a_{n}\right\}$ is a sequence of nonnegative real numbers such that $a_{n+1} \leq\left(1-\gamma_{n}\right) a_{n}+\delta_{n}$, where $\left\{\gamma_{n}\right\}$ is a sequence in $(0,1)$ and $\left\{\delta_{n}\right\}$ is a sequence such that

(1) $\sum_{n=1}^{\infty} \gamma_{n}=\infty$;

(2) $\lim \sup _{n \rightarrow \infty} \delta_{n} / \gamma_{n} \leq 0$ or $\sum_{n=1}^{\infty}\left|\delta_{n}\right|<\infty$.

Then $\lim _{n \rightarrow \infty} a_{n}=0$.

\section{Main results}

Let $H_{1}$ and $H_{2}$ be two real Hilbert spaces. Let $C_{1}, C_{2}, \ldots, C_{N}$ be $N$ nonempty closed convex subsets of $H_{1}$ and let $Q_{1}, Q_{2}, \ldots, Q_{M}$ be $M$ nonempty closed convex subsets of $H_{2}$. Let $A: H_{1} \rightarrow H_{2}$ be a bounded linear operator. Assume that the multiple-sets split feasibility problem is consistent, i.e., it is solvable. Now, we are devoted to solving the constrained multiple-set split feasibility problem (2.2).

For solving (2.2), we introduce the following iterative algorithm.

Algorithm 3.1 Let $f: H_{1} \rightarrow H_{1}$ be a $\rho$-contraction. Let $B: H_{1} \rightarrow H_{1}$ be a self-adjoint, strongly positive bounded linear operator with coefficient $\alpha>0$. Let $\sigma$ and $\gamma$ be two constants such that $0<\gamma<\frac{2}{L}$ and $0<\sigma \rho<\alpha$. For arbitrary initial point $x_{0} \in H_{1}$, we define a sequence $\left\{x_{n}\right\}$ iteratively by

$$
\begin{aligned}
x_{n+1}= & P_{\Omega}\left(I-\gamma\left(\sum_{i=1}^{N} \alpha_{i}\left(I-P_{C_{i}}\right)+\sum_{j=1}^{M} \beta_{j} A^{*}\left(I-P_{Q_{j}}\right) A\right)\right) \\
& \times P_{\Omega}\left(\xi_{n} \sigma f+\left(I-\xi_{n} B\right)\right) x_{n},
\end{aligned}
$$

for all $n \geq 0$, where $\left\{\xi_{n}\right\}$ is a real sequence in $(0,1)$.

Fact 3.2 The mapping $I-\gamma\left(\sum_{i=1}^{N} \alpha_{i}\left(I-P_{C_{i}}\right)+\sum_{j=1}^{M} \beta_{j} A^{*}\left(I-P_{Q_{j}}\right) A\right)$ is $\frac{\gamma L}{2}$-averaged.

In order to check Fact 3.2, we need the following lemmas.

Lemma 3.3 (Baillon-Haddad) [28] If $h: H \rightarrow R$ has an L-Lipschitz continuous gradient $\nabla h$, then $\nabla h$ is $\frac{1}{L}$-ism.

Lemma 3.4 Given $T: H \rightarrow H$ and let $V=I-T$ be the complement of $T$. Given also $S$ : $H \rightarrow H$.

(i) $T$ is nonexpansive if and only if $V$ is $\frac{1}{2}$-inverse strongly monotone (in short, $\frac{1}{2}$-ism).

(ii) If $S$ is $v$-ism, then for $\gamma>0, \gamma S$ is $\frac{\nu}{\gamma}$-ism.

(iii) $S$ is averaged if and only if the complement $I-S$ is $v$-ism for some $v>\frac{1}{2}$.

Lemma 3.5 Given operators $S, T, V: H \rightarrow H$.

(i) If $S=(1-\alpha) T+\alpha V$ for some $\alpha \in(0,1)$ and if $T$ is averaged and $V$ is nonexpansive, then $S$ is averaged. 
(ii) $S$ is firmly nonexpansive if and only if the complement I-S is firmly nonexpansive. If $S$ is firmly nonexpansive, then $S$ is averaged.

(iii) If $S=(1-\alpha) T+\alpha V$ for some $\alpha \in(0,1)$, $T$ is firmly nonexpansive and $V$ is nonexpansive, then $S$ is averaged.

(iv) If $S$ and $T$ are both averaged, then the product (composite) ST is averaged.

Proof of Fact 3.2 Since gradient $\nabla g(x)=\sum_{i=1}^{N} \alpha_{i}\left(I-P_{C_{i}}\right) x+\sum_{j=1}^{M} \beta_{j} A^{*}\left(I-P_{Q_{j}}\right) A x$ has an $L$-Lipschitz constant $L=\sum_{i=1}^{N} \alpha_{i}+\sum_{j=1}^{M} \beta_{j}\|A\|^{2}$, by Lemma 3.4, $\nabla g$ is $\frac{1}{L}$-ism and $\gamma\left(\sum_{i=1}^{N} \alpha_{i}\left(I-P_{C_{i}}\right)+\sum_{j=1}^{M} \beta_{j} A^{*}\left(I-P_{Q_{j}}\right) A\right)$ is $\frac{1}{\gamma L}$-ism. Again, from Lemma 3.4(iii), we deduce that $I-\gamma\left(\sum_{i=1}^{N} \alpha_{i}\left(I-P_{C_{i}}\right)+\sum_{j=1}^{M} \beta_{j} A^{*}\left(I-P_{Q_{j}}\right) A\right)$ is $\frac{\gamma L}{2}$-averaged.

Now, we prove the convergence of the sequence $\left\{x_{n}\right\}$.

Theorem 3.6 Suppose that $S \neq \emptyset$. Assume that the sequence $\left\{\xi_{n}\right\}$ satisfies the control conditions:

(i) $\lim _{n \rightarrow \infty} \xi_{n}=0$ and

(ii) $\sum_{n=0}^{\infty} \xi_{n}=\infty$.

Then the sequence $\left\{x_{n}\right\}$ generated by (3.1) converges to a solution $x^{*}$ of (2.2), where $x^{*}$ also solves the following VI:

$$
x^{*} \in S \text { such that }\left\langle\sigma f\left(x^{*}\right)-B x^{*}, \tilde{x}-x^{*}\right\rangle \leq 0 \quad \text { for all } \tilde{x} \in S
$$

where $S$ is the set of solutions of (2.2).

Proof Let $x^{*} \in S$. Since $B$ is strongly positive bounded linear operator with coefficient $\alpha>0$, we have $\left\|I-\xi_{n} B\right\| \leq 1-\alpha \xi_{n}$ (without loss of generality, we may assume $\xi_{n} \leq \frac{1}{\alpha}$ ). Thus, by (3.1), we have

$$
\begin{aligned}
\left\|x_{n+1}-x^{*}\right\| & \| P_{\Omega}\left(I-\gamma\left(\sum_{i=1}^{N} \alpha_{i}\left(I-P_{C_{i}}\right)+\sum_{j=1}^{M} \beta_{j} A^{*}\left(I-P_{Q_{j}}\right) A\right)\right) \\
& \times P_{\Omega}\left(\xi_{n} \sigma f+\left(I-\xi_{n} B\right)\right) x_{n}-x^{*} \| \\
\leq & \left\|\xi_{n} \sigma f\left(x_{n}\right)+\left(I-\xi_{n} B\right) x_{n}-x^{*}\right\| \\
\leq & \xi_{n} \sigma\left\|f\left(x_{n}\right)-f\left(x^{*}\right)\right\|+\left\|I-\xi_{n} B\right\|\left\|x_{n}-x^{*}\right\|+\xi_{n}\left\|\sigma f\left(x^{*}\right)-B x^{*}\right\| \\
\leq & \xi_{n} \sigma \rho\left\|x_{n}-x^{*}\right\|+\left(1-\xi_{n} \alpha\right)\left\|x_{n}-x^{*}\right\|+\xi_{n}\left\|\sigma f\left(x^{*}\right)-B x^{*}\right\| \\
= & {\left[1-(\alpha-\sigma \rho) \xi_{n}\right]\left\|x_{n}-x^{*}\right\|+(\alpha-\sigma \rho) \xi_{n}\left\|f\left(x^{*}\right)-B x^{*}\right\| /(\alpha-\sigma \rho) . }
\end{aligned}
$$

An induction yields

$$
\begin{aligned}
\left\|x_{n+1}-x^{*}\right\| & \leq \max \left\{\left\|x_{n}-x^{*}\right\|, \frac{\left\|f\left(x^{*}\right)-B x^{*}\right\|}{\alpha-\sigma \rho}\right\} \\
& \leq \max \left\{\left\|x_{0}-x^{*}\right\|, \frac{\left\|f\left(x^{*}\right)-B x^{*}\right\|}{\alpha-\sigma \rho}\right\} .
\end{aligned}
$$

Hence, $\left\{x_{n}\right\}$ is bounded. 
It is well-known that the metric projection $P_{\Omega}$ is firmly nonexpansive, hence averaged. By Fact 3.2, $I-\gamma\left(\sum_{i=1}^{N} \alpha_{i}\left(I-P_{C_{i}}\right)+\sum_{j=1}^{M} \beta_{j} A^{*}\left(I-P_{Q_{j}}\right) A\right)$ is $\frac{\gamma L}{2}$-averaged. From Lemma 3.5, the composite of three averaged mappings is averaged. So, $P_{\Omega}\left(I-\gamma\left(\sum_{i=1}^{N} \alpha_{i}\left(I-P_{C_{i}}\right)+\right.\right.$ $\left.\left.\sum_{j=1}^{M} \beta_{j} A^{*}\left(I-P_{Q_{j}}\right) A\right)\right) P_{\Omega}$ is an averaged mapping. Thus, there must exist a positive constant $\delta \in(0,1)$ such that

$$
P_{\Omega}\left(I-\gamma\left(\sum_{i=1}^{N} \alpha_{i}\left(I-P_{C_{i}}\right)+\sum_{j=1}^{M} \beta_{j} A^{*}\left(I-P_{Q_{j}}\right) A\right)\right) P_{\Omega}=(1-\delta) I+\delta U
$$

where $U$ is a nonexpansive mapping. Set $y_{n}=\xi_{n} \sigma f\left(x_{n}\right)+\left(I-\xi_{n} B\right) x_{n}$ for all $n \geq 0$. Then we have

$$
\begin{aligned}
x_{n+1} & =((1-\delta) I+\delta U)\left(\xi_{n} \sigma f\left(x_{n}\right)+\left(I-\xi_{n} B\right) x_{n}\right) \\
& =(1-\delta) x_{n}+\xi_{n}(1-\delta)\left(\sigma f\left(x_{n}\right)-B x_{n}\right)+\delta U y_{n} \\
& =(1-\delta) x_{n}+\delta\left(\frac{1-\delta}{\delta} \xi_{n}\left(\sigma f\left(x_{n}\right)-B x_{n}\right)+U y_{n}\right) \\
& =(1-\delta) x_{n}+\delta z_{n},
\end{aligned}
$$

where

$$
z_{n}=\frac{(1-\delta) \xi_{n}}{\delta}\left(\sigma f\left(x_{n}\right)-B x_{n}\right)+U y_{n}
$$

By virtue of $\xi_{n} \rightarrow 0($ as $n \rightarrow \infty)$ and the boundedness of the sequences $\left\{f\left(x_{n}\right)\right\}$ and $\left\{B x_{n}\right\}$, we firstly observe that

$$
\lim _{n \rightarrow \infty}\left\|y_{n}-x_{n}\right\|=\lim _{n \rightarrow \infty} \xi_{n}\left\|\sigma f\left(x_{n}\right)-B x_{n}\right\|=0,
$$

and

$$
\lim _{n \rightarrow \infty}\left\|z_{n}-U y_{n}\right\|=\lim _{n \rightarrow \infty} \frac{(1-\delta) \xi_{n}}{\delta}\left\|\sigma f\left(x_{n}\right)-B x_{n}\right\|=0
$$

Next, we estimate $\left\|z_{n+1}-z_{n}\right\|$. Note that

$$
z_{n+1}-z_{n}=\frac{(1-\delta) \xi_{n+1}}{\delta}\left(\sigma f\left(x_{n+1}\right)-B x_{n+1}\right)+U y_{n+1}-\frac{(1-\delta) \xi_{n}}{\delta}\left(\sigma f\left(x_{n}\right)-B x_{n}\right)-U y_{n} .
$$

It follows that

$$
\begin{aligned}
\left\|z_{n+1}-z_{n}\right\| & \leq \frac{1-\delta}{\delta}\left(\xi_{n+1}\left\|\sigma f\left(x_{n+1}\right)-B x_{n+1}\right\|+\xi_{n}\left\|\sigma f\left(x_{n}\right)-B x_{n}\right\|\right)+\left\|U y_{n+1}-U y_{n}\right\| \\
& \leq \frac{1-\delta}{\delta}\left(\xi_{n+1}\left\|\sigma f\left(x_{n+1}\right)-B x_{n+1}\right\|+\xi_{n}\left\|\sigma f\left(x_{n}\right)-B x_{n}\right\|\right)+\left\|y_{n+1}-y_{n}\right\| .
\end{aligned}
$$

Since $y_{n+1}-y_{n}=\xi_{n+1} \sigma f\left(x_{n+1}\right)+\left(I-\xi_{n+1} B\right) x_{n+1}-\xi_{n} \sigma f\left(x_{n}\right)-\left(I-\xi_{n} B\right) x_{n}$, we get

$$
\begin{aligned}
\left\|z_{n+1}-z_{n}\right\| \leq & \left\|\xi_{n+1} \sigma f\left(x_{n+1}\right)+\left(I-\xi_{n+1} B\right) x_{n+1}-\xi_{n} \sigma f\left(x_{n}\right)-\left(I-\xi_{n} B\right) x_{n}\right\| \\
& +\frac{1-\delta}{\delta}\left(\xi_{n+1}\left\|\sigma f\left(x_{n+1}\right)-B x_{n+1}\right\|+\xi_{n}\left\|\sigma f\left(x_{n}\right)-B x_{n}\right\|\right)
\end{aligned}
$$




$$
\begin{aligned}
\leq & \left\|x_{n+1}-x_{n}\right\|+\xi_{n+1}\left\|\sigma f\left(x_{n+1}\right)-B x_{n+1}\right\|+\xi_{n}\left\|\sigma f\left(x_{n}\right)-B x_{n}\right\| \\
& +\frac{1-\delta}{\delta}\left(\xi_{n+1}\left\|\sigma f\left(x_{n+1}\right)-B x_{n+1}\right\|+\xi_{n}\left\|\sigma f\left(x_{n}\right)-B x_{n}\right\|\right) .
\end{aligned}
$$

It follows that

$$
\begin{aligned}
\left\|z_{n+1}-z_{n}\right\|-\left\|x_{n+1}-x_{n}\right\| \leq & \xi_{n+1}\left\|\sigma f\left(x_{n+1}\right)-B x_{n+1}\right\|+\xi_{n}\left\|\sigma f\left(x_{n}\right)-B x_{n}\right\| \\
& +\frac{1-\delta}{\delta}\left(\xi_{n+1}\left\|\sigma f\left(x_{n+1}\right)-B x_{n+1}\right\|+\xi_{n}\left\|\sigma f\left(x_{n}\right)-B x_{n}\right\|\right) .
\end{aligned}
$$

Since $\lim _{n \rightarrow \infty} \xi_{n}=0$ and the sequences $\left\{f\left(x_{n}\right)\right\},\left\{B x_{n}\right\}$ are bounded, we deduce

$$
\limsup _{n \rightarrow \infty}\left(\left\|z_{n+1}-z_{n}\right\|-\left\|x_{n+1}-x_{n}\right\|\right) \leq 0
$$

By Lemma 2.1, we get

$$
\lim _{n \rightarrow \infty}\left\|z_{n}-x_{n}\right\|=0
$$

Therefore,

$$
\begin{aligned}
& \lim _{n \rightarrow \infty}\left\|U x_{n}-x_{n}\right\| \\
& \quad=\lim _{n \rightarrow \infty}\left\|P_{\Omega}\left(I-\gamma\left(\sum_{i=1}^{N} \alpha_{i}\left(I-P_{C_{i}}\right)+\sum_{j=1}^{M} \beta_{j} A^{*}\left(I-P_{Q_{j}}\right) A\right)\right) P_{\Omega}\left(x_{n}\right)-x_{n}\right\|=0 .
\end{aligned}
$$

By the definition of the sequence $\left\{x_{n}\right\}$, we know that $x_{n} \in \Omega$. Hence, $P_{\Omega}\left(x_{n}\right)=x_{n}$. So,

$$
\lim _{n \rightarrow \infty}\left\|P_{\Omega}\left(I-\gamma\left(\sum_{i=1}^{N} \alpha_{i}\left(I-P_{C_{i}}\right)+\sum_{j=1}^{M} \beta_{j} A^{*}\left(I-P_{Q_{j}}\right) A\right)\right) x_{n}-x_{n}\right\|=0
$$

Next we prove

$$
\limsup _{n \rightarrow \infty}\left\langle\sigma f\left(x^{*}\right)-B x^{*}, P_{\Omega}\left(y_{n}\right)-x^{*}\right\rangle \leq 0
$$

In order to get this inequality, we need to prove the following:

$$
\limsup _{n \rightarrow \infty}\left\langle\sigma f\left(x^{*}\right)-B x^{*}, x_{n}-x^{*}\right\rangle \leq 0
$$

where $x^{*}$ is the unique solution of VI(3.2). For this purpose, we choose a subsequence $\left\{x_{n_{i}}\right\}$ of $\left\{x_{n}\right\}$ such that

$$
\limsup _{n \rightarrow \infty}\left\langle\sigma f\left(x^{*}\right)-B x^{*}, x_{n}-x^{*}\right\rangle=\lim _{i \rightarrow \infty}\left\langle\sigma f\left(x^{*}\right)-B x^{*}, x_{n_{i}}-x^{*}\right\rangle
$$

Since $\left\{x_{n_{i}}\right\}$ is bounded, there exists a subsequence of $\left\{x_{n_{i}}\right\}$ which converges weakly to a point $\tilde{x}$. Without loss of generality, we may assume that $\left\{x_{n_{i}}\right\}$ converges weakly to $\tilde{x}$. Since 
$P_{\Omega}\left(I-\gamma\left(\sum_{i=1}^{N} \alpha_{i}\left(I-P_{C_{i}}\right)+\sum_{j=1}^{M} \beta_{j} A^{*}\left(I-P_{Q_{j}}\right) A\right)\right)$ is nonexpansive, by Lemma 2.2 , we have $x_{n_{i}} \rightarrow \tilde{x} \in \operatorname{Fix}\left(P_{\Omega}\left(I-\gamma\left(\sum_{i=1}^{N} \alpha_{i}\left(I-P_{C_{i}}\right)+\sum_{j=1}^{M} \beta_{j} A^{*}\left(I-P_{Q_{j}}\right) A\right)\right)\right)$. Therefore,

$$
\begin{aligned}
\limsup _{n \rightarrow \infty}\left\langle\sigma f\left(x^{*}\right)-B x^{*}, x_{n}-x^{*}\right\rangle & =\lim _{i \rightarrow \infty}\left\langle\sigma f\left(x^{*}\right)-B x^{*}, x_{n_{i}}-x^{*}\right\rangle \\
& =\left\langle\sigma f\left(x^{*}\right)-B x^{*}, \tilde{x}-x^{*}\right\rangle \leq 0 .
\end{aligned}
$$

Since $\left\|x_{n}-P_{\Omega}\left(y_{n}\right)\right\|=\left\|P_{\Omega}\left(x_{n}\right)-P_{\Omega}\left(y_{n}\right)\right\| \leq\left\|x_{n}-y_{n}\right\| \rightarrow 0$, we obtain

$$
\limsup _{n \rightarrow \infty}\left\langle\sigma f\left(x^{*}\right)-B x^{*}, P_{\Omega}\left(y_{n}\right)-x^{*}\right\rangle \leq 0
$$

Note that

$$
\left\|P_{\Omega}\left(y_{n}\right)-x^{*}\right\|^{2}=\left\langle P_{\Omega}\left(y_{n}\right)-y_{n}, P_{\Omega}\left(y_{n}\right)-x^{*}\right\rangle+\left\langle y_{n}-x^{*}, P_{\Omega}\left(y_{n}\right)-x^{*}\right\rangle .
$$

From the property of the metric $P_{\Omega}$, we have $\left\langle P_{\Omega}\left(y_{n}\right)-y_{n}, P_{\Omega}\left(y_{n}\right)-x^{*}\right\rangle \leq 0$. Hence,

$$
\begin{aligned}
\left\|P_{\Omega}\left(y_{n}\right)-x^{*}\right\|^{2} \leq & \left\langle y_{n}-x^{*}, P_{\Omega}\left(y_{n}\right)-x^{*}\right\rangle \\
= & \left\langle\xi_{n} \sigma\left(f\left(x_{n}\right)-f\left(x^{*}\right)\right)+\left(I-\xi_{n} B\right)\left(x_{n}-x^{*}\right), P_{\Omega}\left(y_{n}\right)-x^{*}\right\rangle \\
& +\xi_{n}\left\langle\sigma f\left(x^{*}\right)-B x^{*}, P_{\Omega}\left(y_{n}\right)-x^{*}\right\rangle \\
\leq & \left(\xi_{n} \sigma\left\|f\left(x_{n}\right)-f\left(x^{*}\right)\right\|+\left\|I-\xi_{n} B\right\|\left\|x_{n}-x^{*}\right\|\right)\left\|P_{\Omega}\left(y_{n}\right)-x^{*}\right\| \\
& +\xi_{n}\left\langle\sigma f\left(x^{*}\right)-B x^{*}, P_{\Omega}\left(y_{n}\right)-x^{*}\right\rangle \\
\leq & \left(1-\xi_{n}(\alpha-\sigma \rho)\right)\left\|x_{n}-x^{*}\right\|\left\|P_{\Omega}\left(y_{n}\right)-x^{*}\right\| \\
& +\xi_{n}\left\langle\sigma f\left(x^{*}\right)-B x^{*}, P_{\Omega}\left(y_{n}\right)-x^{*}\right\rangle \\
\leq & \frac{1-\xi_{n}(\alpha-\sigma \rho)}{2}\left\|x_{n}-x^{*}\right\|^{2}+\frac{1}{2}\left\|P_{\Omega}\left(y_{n}\right)-x^{*}\right\|^{2} \\
& +\xi_{n}\left\langle\sigma f\left(x^{*}\right)-B x^{*}, P_{\Omega}\left(y_{n}\right)-x^{*}\right\rangle .
\end{aligned}
$$

It follows that

$$
\left\|P_{\Omega}\left(y_{n}\right)-x^{*}\right\|^{2} \leq\left[1-(\alpha-\sigma \rho) \xi_{n}\right]\left\|x_{n}-x^{*}\right\|^{2}+2 \xi_{n}\left\langle\sigma f\left(x^{*}\right)-B x^{*}, P_{\Omega}\left(y_{n}\right)-x^{*}\right\rangle .
$$

Finally, we show that $x_{n} \rightarrow x^{*}$. From (3.1), we have

$$
\begin{aligned}
\left\|x_{n+1}-x^{*}\right\|^{2}= & \left\|P_{\Omega}\left(I-\gamma\left(\sum_{i=1}^{N} \alpha_{i}\left(I-P_{C_{i}}\right)+\sum_{j=1}^{M} \beta_{j} A^{*}\left(I-P_{Q_{j}}\right) A\right)\right) P_{\Omega}\left(y_{n}\right)-x^{*}\right\|^{2} \\
\leq & \left\|P_{\Omega}\left(y_{n}\right)-x^{*}\right\|^{2} \\
\leq & {\left[1-(\alpha-\sigma \rho) \xi_{n}\right]\left\|x_{n}-x^{*}\right\|^{2} } \\
& +(\alpha-\sigma \rho) \xi_{n} \frac{2}{\alpha-\sigma \rho}\left\langle\sigma f\left(x^{*}\right)-B x^{*}, P_{\Omega}\left(y_{n}\right)-x^{*}\right\rangle \\
= & \left(1-\gamma_{n}\right)\left\|x_{n}-x^{*}\right\|^{2}+\delta_{n},
\end{aligned}
$$


where $\gamma_{n}=(\alpha-\sigma \rho) \xi_{n}$ and $\delta_{n}=(\alpha-\sigma \rho) \xi_{n} \frac{2}{\alpha-\sigma \rho}\left\langle\sigma f\left(x^{*}\right)-B x^{*}, P_{\Omega}\left(y_{n}\right)-x^{*}\right\rangle$. Since $\sum_{n=1}^{\infty} \gamma_{n}=$ $\infty$ and $\limsup _{n \rightarrow \infty} \frac{\delta_{n}}{\gamma_{n}}=\limsup _{n \rightarrow \infty} \frac{2}{\alpha-\sigma \rho}\left\langle\sigma f\left(x^{*}\right)-B x^{*}, P_{\Omega}\left(y_{n}\right)-x^{*}\right\rangle \leq 0$, all conditions of Lemma 2.3 are satisfied. Therefore, we immediately deduce that $x_{n} \rightarrow x^{*}$. This completes the proof.

From (3.1) and Theorem 3.6, we can deduce easily the following results.

Algorithm 3.7 For an arbitrary initial point $x_{0} \in H_{1}$, we define a sequence $\left\{x_{n}\right\}$ iteratively by

$$
\begin{aligned}
x_{n+1}= & P_{\Omega}\left(I-\gamma\left(\sum_{i=1}^{N} \alpha_{i}\left(I-P_{C_{i}}\right)+\sum_{j=1}^{M} \beta_{j} A^{*}\left(I-P_{Q_{j}}\right) A\right)\right) \\
& \times P_{\Omega}\left(\xi_{n} \sigma f\left(x_{n}\right)+\left(1-\xi_{n}\right) x_{n}\right),
\end{aligned}
$$

for all $n \geq 0$, where $\left\{\xi_{n}\right\}$ is a real sequence in $(0,1)$.

Corollary 3.8 Suppose that $S \neq \emptyset$. Assume that the sequence $\left\{\xi_{n}\right\}$ satisfies the conditions

(i) $\lim _{n \rightarrow \infty} \xi_{n}=0$ and

(ii) $\sum_{n=0}^{\infty} \xi_{n}=\infty$.

Then the sequence $\left\{x_{n}\right\}$ generated by (3.3) converges to a point $x^{*}$, which solves the following variational inequality:

$$
x^{*} \in S \text { such that }\left\langle\sigma f\left(x^{*}\right)-x^{*}, \tilde{x}-x^{*}\right\rangle \leq 0 \quad \text { for all } \tilde{x} \in S
$$

Algorithm 3.9 For an arbitrary initial point $x_{0}$, we define a sequence $\left\{x_{n}\right\}$ iteratively by

$$
x_{n+1}=P_{\Omega}\left(I-\gamma\left(\sum_{i=1}^{N} \alpha_{i}\left(I-P_{C_{i}}\right)+\sum_{j=1}^{M} \beta_{j} A^{*}\left(I-P_{Q_{j}}\right) A\right)\right) P_{\Omega}\left(\left(1-\xi_{n}\right) x_{n}\right) \text {, }
$$

for all $n \geq 0$, where $\left\{\xi_{n}\right\}$ is a real sequence in $(0,1)$.

Corollary 3.10 Suppose that $S \neq \emptyset$. Assume that the sequence $\left\{\xi_{n}\right\}$ satisfies the conditions

(i) $\lim _{n \rightarrow \infty} \xi_{n}=0$ and

(ii) $\sum_{n=0}^{\infty} \xi_{n}=\infty$.

Then the sequence $\left\{x_{n}\right\}$ generated by (3.4) converges to a point $x^{*} \in S$ which is the minimum norm element in $S$.

\section{Competing interests}

The authors declare that they have no competing interests.

\section{Authors' contributions}

All authors read and approved the final manuscript.

\section{Author details}




\section{Acknowledgements}

Jinwei Shi was supported in part by the scientific research fund of the Educational Commission of Hebei Province of China (No. 936101101) and the National Natural Science Foundation of China (No. 51077053). Yeong-Cheng Liou was supported in part by NSC 101-2628-E-230-001-MY3 and NSC 101-2622-E-230-005-CC3.

Received: 30 January 2013 Accepted: 14 May 2013 Published: 31 May 2013

\section{References}

1. Censor, Y, Elfving, T: A multiprojection algorithm using Bregman projections in a product space. Numer. Algorithms 8 , 221-239 (1994)

2. Byrne, C: Iterative oblique projection onto convex subsets and the split feasibility problem. Inverse Probl. 18, 441-453 (2002)

3. Censor, Y, Elfving, T, Kopf, N, Bortfeld, T: The multiple-sets split feasibility problem and its applications for inverse problems. Inverse Probl. 21, 2071-2084 (2005)

4. Censor, Y, Motova, A, Segal, A: Perturbed projections and subgradient projections for the multiple-sets split feasibility problem. J. Math. Anal. Appl. 327, 1244-1256 (2007)

5. Censor, Y, Bortfeld, T, Martin, B, Trofimov, A: A unified approach for inversion problems in intensity modulated radiation therapy. Phys. Med. Biol. 51, 2353-2365 (2006)

6. Xu, HK: A variable Krasnosel'skii-Mann algorithm and the multiple-set split feasibility problem. Inverse Probl. 22 2021-2034 (2006)

7. $\mathrm{Xu}, \mathrm{HK}$ : Iterative methods for the split feasibility problem in infinite-dimensional Hilbert spaces. Inverse Probl. 26, $105018(2010)$

8. Yao, Y, Chen, R, Marino, G, Liou, YC: Applications of fixed point and optimization methods to the multiple-sets split feasibility problem. J. Appl. Math. 2012, Article ID 927530 (2012)

9. Yao, Y, Wu, J, Liou, YC: Regularized methods for the split feasibility problem. Abstr. Appl. Anal. 2012, Article ID 140679 (2012). doi:10.1155/2012/140679

10. Yao, Y, Yang, PX, Kang, SM: Composite projection algorithms for the split feasibility problem. Math. Comput. Model. 57, 693-700 (2013)

11. Byrne, C: An unified treatment of some iterative algorithm algorithms in signal processing and image reconstruction Inverse Probl. 20, 103-120 (2004)

12. Qu, B, Xiu, N: A note on the CQ algorithm for the split feasibility problem. Inverse Probl. 21, 1655-1665 (2005)

13. Yang, Q: The relaxed CQ algorithm solving the split feasibility problem. Inverse Probl. 20, 1261-1266 (2004)

14. Zhao, J, Yang, Q: Several solution methods for the split feasibility problem. Inverse Probl. 21, 1791-1799 (2005)

15. Wang, $F, X u, H K$ : Approximating curve and strong convergence of the $C Q$ algorithm for the split feasibility problem. J. Inequal. Appl. (2010). doi:10.1155/2010/102085

16. Dang, Y, Gao, Y: The strong convergence of a KM-CQ-like algorithm for a split feasibility problem. Inverse Probl. 27, $015007(2011)$

17. Yu, X, Shahzad, N, Yao, Y: Implicit and explicit algorithms for solving the split feasibility problem. Optim. Lett. 6, 1447-1462 (2012)

18. Dang, Y, Gao, Y, Han, Y: A perturbed projection algorithm with inertial technique for split feasibility problem. J. Appl. Math. 2012, Article ID 207323 (2012)

19. Dang, Y, Gao, Y: An extrapolated iterative algorithm for multiple-set split feasibility problem. Abstr. Appl. Anal. 2012, Article ID 149508 (2012)

20. Dang, Y, Gao, Y: The strong convergence of a KM-CQ-like algorithm for a split feasibility problem. Inverse Probl. 27(1), Article ID 015007 (2011)

21. Yao, Y, Kim, TH, Chebbi, S, Xu, HK: A modified extragradient method for the split feasibility and fixed point problems. J. Nonlinear Convex Anal. 13(3), 383-396 (2012)

22. Wang, Z, Yang, Q, Yang, Y: The relaxed inexact projection methods for the split feasibility problem. Appl. Math Comput. (2010). doi:10.1016/j.amc.2010.11.058

23. Ceng, LC, Yao, JC: Relaxed and hybrid viscosity methods for general system of variational inequalities with split feasibility problem constraint. Fixed Point Theory Appl. 2013, Article ID 43 (2013)

24. Ceng, LC, Ansari, QH, Yao, JC: Relaxed extragradient methods for finding minimum-norm solutions of the split feasibility problem. Nonlinear Anal., Theory Methods Appl. 75, 2116-2125 (2012)

25. Suzuki, T: Strong convergence theorems for infinite families of nonexpansive mappings in general Banach spaces. Fixed Point Theory Appl. 2005, 103-123 (2005)

26. Geobel, K, Kirk, WA: Topics in Metric Fixed Point Theory. Cambridge Studies in Advanced Mathematics, vol. 28 Cambridge University Press, Cambridge (1990)

27. Xu, HK: Iterative algorithms for nonlinear operators. J. Lond. Math. Soc. 66, 240-256 (2002)

28. Baillon, JB, Haddad, G: Quelques proprietes des operateurs anglebornes et $n$-cycliquement monotones. Isr. J. Math. $26,137-150(1977)$

doi:10.1186/1029-242X-2013-272

Cite this article as: Zheng et al.: The constrained multiple-sets split feasibility problem and its projection algorithms. Journal of Inequalities and Applications 2013 2013:272. 